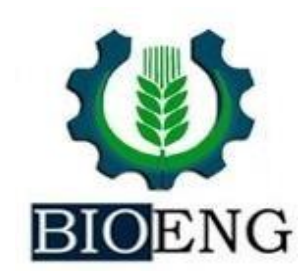

\title{
ANÁLISE DAS VARIÁVEIS AMBIENTAIS E FISIOLÓGICAS DE AVES POEDEIRAS COM E SEM ENRIQUECIMENTO AMBIENTAL
}

\author{
A. P. Ribeiro", L. F. Silva ${ }^{2 *}$, I. Menegali, F ${ }^{1}$. Ferreira $^{1}$
}

${ }^{1}$ Instituto de Ciências Agrárias da Universidade Federal de Minas Gerais ICA-UFMG, Av. Universitária, 1000 - Universitário, Montes Claros, Minas Gerais, Brasil - MG, 39404-547

${ }^{2}$ Universidade Federal de Viçosa, Departamento de Engenharia Agrícola, Viçosa, Minas Gerais, Brasil

Article history: Received 16 August; Received in revised form 04 December 2020; Accepted 04 December 2020; Available online 30 December 2020.

\section{RESUMO}

Objetivou-se, verificar a influência dos tratamentos com e sem enriquecimento ambiental sobre o comportamento de poedeiras e a qualidade dos ovos, bem como estudar as variáveis térmicas temperatura e umidade durante os horários em que foram avaliados o comportamento de interação das aves com o enriquecimento empregado. O experimento foi conduzido em delineamento em blocos casualizados (DBC), com dois tratamentos (gaiolas convencionais com enriquecimento e gaiolas convencionais sem enriquecimento) e 18 repetições por tratamento, duas aves por repetição, durante 28 dias. Os dados coletados no experimento foram analisados com auxílio do programa para análises estatística e genéticas - SAEG (SAEG, 2009), por meio de análise de variância e submetidos a teste Fisher a 5\% probabilidade para detectar diferença significativa existente entre os tratamentos avaliados para variáveis ambientais e fisiológicas mensuradas. Para análise do comportamento das aves, os dados foram analisados por meio do Teste de $\chi^{2}$. De acordo com as temperaturas aferidas nos sensores, houve uma diferença evidente entre os horários de coleta dos dados. Não houve efeito significativo dos tratamentos sobre as características de peso dos ovos, altura de albúmen, índice de gema, espessura de casca e Unidade Haugh. Não foi observado efeito dos blocos laterais na temperatura retal, porém houve um efeito significativo para blocos sobre as variáveis temperatura média da pele e temperatura média corporal.

Palavras-Chave: Ambiente térmico, avicultura de postura, bem-estar, enriquecimento ambiental

\section{EVALUATION OF ENVIRONMENTAL AND PHYSIOLOGICAL VARIABLES OF HY- LINE BROWN POULTRY WITH AND WITHOUT ENVIRONMENTAL ENRICHMENT}

\begin{abstract}
The objective was to verify the influence of treatments with and without environmental enrichment on the behavior of layers and egg quality, as well as to study the thermal variables temperature and humidity during the times when the interaction behavior of birds with enrichment was evaluated employee. Also evaluating the thermal variables of air temperature and relative humidity. The experiment was conducted in a randomized complete block design (DBC) with two treatments (conventional enrichment cages and conventional cages without enrichment) and 18 replicates per
\end{abstract}

\footnotetext{
*franca.leonardo@yahoo.com.br
} 
treatment, two birds per replicate, for 28 days. The data collected in the experiment were analyzed using a statistical and genetic analysis program (SAEG, 2009), using analysis of variance and subjected to Fisher test at 5\% probability to detect the significant difference between treatments evaluated for variables environmental and physiological parameters. To analyze the behavior of the birds, the data were analyzed by means of the $\chi^{2}$ Test. According to the temperatures measured in the sensors, there was an evident difference between the times of data collection. There was no significant effect of treatments on egg weight characteristics, albumen height, yolk index, shell thickness, and Haugh Unit. No effect of the lateral blocks on the rectal temperature was observed, but there was a significant effect for blocks on the variables mean of skin temperature and mean body temperature

Key words: Thermal environment, aviculture of posture, well-being, environmental enrichment.

\section{INTRODUÇÃO}

Atualmente a preocupação com a alimentação e estilo saudável de vida vem aumentando cada dia mais entre a população. A origem dos alimentos e como estes são produzidos, sejam eles de origem vegetal ou animal, tem sido questionado pelos consumidores. Em relação aos alimentos de origem animal, envolve não somente a proveniência do produto final, mas também a forma de criação e os métodos utilizados para a produção destes produtos, principalmente em produções de grande porte.

O bem-estar animal dentro deste contexto é uma preocupação que acomete grande parte dos consumidores em geral. Diante disso, produtores buscam novas formas para um melhor desempenho produtivo dos animais, com o intuito de amenizar o estresse, proporcionando melhores condições de conforto e de desenvolvimento dos mesmos (ANDRADE et al., 2017; CAMERINI, et al., 2016; CASTILHO, et al., 2015; YILDIRIM, TASKIN, 2017).

Para Azevedo et al., (2016) e Souza et al., (2017), essa preocupação com conforto e bemestar animal na avicultura de postura vem crescendo no país, pois a produção avícola em escala industrial define sistemas de criação nos quais as aves são alojadas em gaiolas durante quase toda vida ou dependendo do sistema adotado todo o seu ciclo produtivo,

\section{MATERIAL E MÉTODOS}

O estudo foi realizado no galpão para aves poedeiras, da Fazenda Experimental Professor principalmente na fase de produção de ovos.

Segundo o Relatório Anual da Associação Brasileira de Proteína Animal, ABPA (2019) $99,57 \%$ de toda a produção brasileira de ovos em 2016, que foi pouco menor quer 39,2 bilhões de unidades teve como finalidade $o$ abastecimento interno do país, tendo um consumo de ovos no brasil de 190 unidades per capita.

De acordo com o relatório (2019) esse valor total de produção foi de quase $40 \%$ superior ao ano de 2010 e com perspectiva de aumento para o ano de 2018. Ainda de acordo com a ABPA (2017) o Estado de Minas Gerais possui o segundo maior alojamento de pintainhas com $11,31 \%$, ficando atrás apenas do estado de São Paulo com 33,09\%.

Com relação a exportação Minas Gerais tem o maior percentual, com $40,09 \%$, de sua produção para o abastecimento de outros países. Quanto à exportação, 84\% são de ovos in natura tendo como maior importador os Emirados Árabes unidos.

Objetivou-se, analisar a influência dos tratamentos com e sem enriquecimento ambiental das gaiolas sobre o comportamento de poedeiras e a qualidade dos ovos, bem como estudar as variáveis térmicas temperatura e umidade durante os horários em que foram avaliados o comportamento de interação das aves com o enriquecimento empregado.

Hamilton de Abreu Navarro (FEHAN), no Instituto de Ciências Agrárias (ICA) da 
Universidade Federal de Minas Gerais. O galpão utilizado possui as seguintes dimensões: 20,2 m de comprimento por 5,5 m de largura, pé direito de $3,56 \mathrm{~m}$, com quatro fileiras de gaiolas metálicas, dispostas em esquema convencional. Cada gaiola com dimensões $40 \mathrm{~cm}$ de profundidade, $38 \mathrm{~cm}$ de altura e $50 \mathrm{~cm}$ de comprimento.

As aves da linhagem Hy-line brown iniciaram o experimento com 58 semanas de idade, e receberam a mesma dieta durante todo o período experimental, e foto período de 16 horas de luz. As gaiolas dispunham de bebedouro tipo nipple e comedouro tipo calha em chapa galvanizada.

$\mathrm{O}$ experimento foi conduzido em delineamento em blocos casualizados (DBC), com dois tratamentos (gaiolas convencionais com enriquecimento e gaiolas convencionais sem enriquecimento) e 18 repetições por tratamento, duas aves por repetição, durante 28 dias.

Os tipos de enriquecimentos utilizados foram chocalhos metálicos, chocalhos de PVC e poleiros, inseridos nas gaiolas que compunham $\mathrm{o}$ tratamento com enriquecimento. $\mathrm{O}$ comportamento das aves com relação a interação com os enriquecimentos ou não, foram observados durante um minuto cada e avaliados diariamente em três horários (10; 15 e 18:30h, conforme o preconizado por Guinzelli, Battiston (2018) e Sans et al.,(2014).

Uma das formas de mensurar o estresse térmico é a utilização de variáveis de conforto como o a Temperatura e Umidade (Menegali,

\section{RESULTADOS E DISCUSSÃO}

O comportamento de interação das poedeiras com os enriquecimentos ambientais nas gaiolas não apresentaram efeito significativos pelo teste $\chi^{2}$ entre os valores observados e esperados dentro dos horários
2009). Sendo assim, as variáveis ambientais como temperatura e umidade foram mensuradas em três horários diariamente (10; 15 e 18:30h) durante todo o período experimental (28 dias), por meio de sensores conectados a mini datalogger localizado no interior da instalação, a altura das aves, e fora da instalação representando o microclima do local. Portanto, para análise estatística dessas variáveis ambientais utilizou-se a média do período experimental em cada horário de mensuração.

Ao final de cada semana, foram mensuradas variáveis fisiológicas como temperatura média de pele (TMP) e temperatura média corporal (TMC); qualidade interna e externa dos ovos (altura de albúmen, altura de gema, diâmetro de gema, unidade Haugh, gravidade especifica e espessura da casca), em cada tratamento.

Os dados coletados no experimento foram analisados com auxílio do programa para análises estatística e genéticas - SAEG (SAEG, 2009), por meio de análise de variância, submetidos ao teste Fisher a 5\% probabilidade e teste de Tukey a 5\% probabilidade para detectar diferença significativa existente entre os tratamentos avaliados para variáveis ambientais e fisiológicas mensuradas.

Para análise do comportamento das aves, os dados foram analisados por meio do Teste de $\chi^{2}$, considerando-se a relação entre a interação da ave com o enriquecimento ou não, conforme o estabelecido Pereira et al., (2013) e Sans et al.,(2014).

considerados (Tabela 1), divergindo do que relataram Abreu et al. (2006) em suas pesquisas sobre enriquecimento ambiental de gaiolas como estratégia prática para incrementar o bemestar e a produção de ovos de poedeiras pesadas. 
Tabela 1 - Interação das aves em relação aos enriquecimentos durante os horários analisados diariamente $(10 ; 15$ e $18: 30 h)$ durante o período experimental.

\begin{tabular}{rccccc}
\hline & & $\mathbf{1 0 : 0 0} \mathbf{h}$ & $\mathbf{1 5 : 0 0} \mathbf{h}$ & $\mathbf{1 8 : 3 0 ~}$ & Total \\
\hline \multirow{2}{*}{ Não } & $\mathrm{n}$ & 98 & 101 & 94 & 293 \\
\cline { 2 - 6 } interagiu & $\mathrm{N}$ & 97,67 & 97,67 & 97,67 & - \\
& $\%$ & 60,5 & 62,3 & 58 & 60,29 \\
\hline \multirow{2}{*}{ Interagiu } & $\mathrm{n}$ & 64 & 61 & 68 & 193 \\
& $\mathrm{~N}$ & 64,33 & 64,33 & 64,33 & - \\
\hline \multirow{2}{*}{ Total } & $\%$ & 39,5 & 37,7 & 42 & 39,71 \\
\hline & $\mathrm{n}$ & 162 & 162 & 162 & 486 \\
& $\%$ & 33,3 & 33,3 & 33,3 & 100 \\
\hline
\end{tabular}

$\mathrm{n}$ - frequência observada; $\mathrm{N}$ - frequência esperada $\chi^{2} ; \%$ - porcentagem em relação ao total observado.

Alguns aspectos podem ter interferido no presente estudo, como temperatura e umidade do ar e os horários de avaliação, como reportado por Pereira et al., (2013), sobre comportamento de poedeiras criadas em diferentes densidades e tamanhos de grupo em ambiente enriquecido, também utilizando o teste $\chi^{2}$ para os comportamentos de empoleirar, os quais verificaram baixa ocorrência do comportamento de empoleirar em horários entre 9 e $10 \mathrm{~h}$ e 15 e $16 \mathrm{~h}$ durante os períodos de observação, uma vez que espera-se maior incidência de empoleiramento ao entardecer.

$\mathrm{O}$ fato dos enriquecimentos ambientais, objetos inseridos nas gaiolas das aves, serem desconhecidos por elas, ainda que seja um comportamento natural como o de empoleirar, pode ter sido uma influência sobre o seu uso, já que não houve um período de adaptação para as mesmas antes das observações para o experimento.

Pode-se inferir também ao comportamento predominante de não interagir com os enriquecimentos dispostos nas gaiolas, em todos os horários avaliados por estar associado a época do ano, onde as temperaturas médias encontraram-se elevadas e baixa umidade relativa do ar, como pode ser observado na (Tabela 2) contendo as médias internas da instalação de temperatura e umidade, temperatura externa e umidade externa da instalação, de acordo com os horários de avaliação do comportamento das aves durante o experimento (10, 15 e 18:30 h).

Tabela 2: Médias das variáveis de acordo com os horários de avaliação do comportamento das aves durante o experimento

\begin{tabular}{llll}
\hline VARIÁVEIS AMBIENTAIS & $\mathbf{1 0 : 0 0 ~ h}$ & $\mathbf{1 5 : 0 0 ~ h}$ & $\mathbf{1 8 : 3 0 ~ h}$ \\
\hline Temperatura do ar interna & $24,325 \mathrm{a}$ & $27,427 \mathrm{a}$ & $26,098 \mathrm{~b}$ \\
\hline Umidade do ar interna & $82,122 \mathrm{a}$ & $79,044 \mathrm{ab}$ & $73,881 \mathrm{~b}$ \\
Temperatura do ar externa & $25,425 \mathrm{a}$ & $28,529 \mathrm{~b}$ & $26,625 \mathrm{~b}$ \\
Umidade do ar externa & $76,296 \mathrm{a}$ & $65,051 \mathrm{ab}$ & $69,751 \mathrm{~b}$ \\
\hline
\end{tabular}

Médias seguidas por letras distintas na linha diferem entre si pelo teste Tukey a $5 \%$ probabilidade

De acordo com as temperaturas aferidas nos sensores, houve uma diferença evidente entre os horários de coleta dos dados. Para Barbosa Filho (2004), diversos trabalhos já publicados afirmam que existe uma faixa adequada de temperatura para o conforto térmico das aves poedeiras, e a interação de variáveis como umidade relativa do ar.

Essas condições climáticas, temperatura e umidade, podem comumente desencadear nas aves o aumento de frequência respiratória e a redução em sua movimentação dentro da gaiola. Também podem interferir na qualidade dos ovos produzido quando as aves estão sob estresse por calor, com queda na produção de ovos e a ocorrência de ovos com casca mole (BARBOSA FILHO, 2004).

Carvalho e Fernandes (2013) afirmam que em temperaturas acima de $26^{\circ} \mathrm{C}$, ou seja, superior ao nível de conforto térmico percebese queda na qualidade da casca.

Quando as temperaturas estão acima dos 
níveis favoráveis as condições de bem-estar, as aves começam um processo de tentativa termo regulatório, ou seja, tentativa de perda de calor, descrito pela hiperventilação e evaporação de água pelos pulmões, levando a alteração no equilíbrio ácido-básico das aves, causando assim danos na formação dos ovos.

Associada diretamente ao bem-estar das aves, tanto por estresse do ambiente de alojamento quanto a fatores ambientais, temperatura do ar e umidade relativa do ar, foram analisados durante o experimento para inferir sobre a qualidade dos ovos.
Os dados foram coletados durante as 4 semanas experimentais, sendo esta avaliação realizada 1 vez por semana, efetuou-se a média dessas semanas, de cada variável, e utilizou-se o teste de Fisher a 5\% probabilidade, para as comparações entre os tratamentos com enriquecimento e sem enriquecimento. Avaliou-se peso do ovo (PO), gravidade específica (GE), altura de albúmen (AA), índice de gema (IG), espessura da casca (ESP), unidade Haugh (UH), de acordo com os tratamentos empregados (Tabela 3 ).

Tabela 3: Médias das variáveis para qualidade dos ovos

\begin{tabular}{ccccccc}
\hline TRATAMENTO & PO & GE & AA & IG & ESP & UH \\
\hline Sem enriquecimento & $61,021 \mathrm{a}$ & $1,091 \mathrm{~b}$ & $9,143 \mathrm{a}$ & $0,467 \mathrm{a}$ & $0,601 \mathrm{a}$ & $95,009 \mathrm{a}$ \\
Com enriquecimento & $60,651 \mathrm{a}$ & $1,094 \mathrm{a}$ & $9,406 \mathrm{a}$ & $0,469 \mathrm{a}$ & $0,601 \mathrm{a}$ & $96,345 \mathrm{a}$ \\
\hline
\end{tabular}

Médias seguidas por letras distintas na coluna diferem entre si pelo teste Fisher a 5\% probabilidade

Não houve efeito significativo dos tratamentos sobre as características de peso dos ovos, altura de albúmen, índice de gema, espessura de casca e Unidade Haugh. Entretanto, para gravidade específica dos ovos foi observado diferença significativa pelo teste Fisher 5\% probabilidade, onde os ovos produzidos pelas aves submetidas ao tratamento com enriquecimento apresentaram melhor gravidade específica.

Em suas pesquisas sobre a comparação de métodos de determinação de gravidade específica, os autores Freitas et al. (2004) citam que a gravidade especifica está ligada diretamente ao percentual da casca, sendo capaz de ser empregada como método indireto na determinação da qualidade da casca. Os autores ainda afirmam que a cada acréscimo de 0,001 na GE, a porcentagem de ovos quebrados decresceu em $1,266 \%$.

A mensuração das temperaturas corporais nas aves, é uma das características que auxiliam nos parâmetros de bem-estar e estresse das poedeiras. O local de instalação do galpão com relação ao posicionamento do sol, exerce grande influência sobre as temperaturas no interior no galpão, já que pode haver oscilações de temperaturas devido a variação de incidência solar nas laterais da estrutura.

Para Cordeiro et al. (2014) os fatores climáticos são extremamente importantes por influenciar diretamente o conforto e bem-estar das aves, e tendo ligação direta a produção avícola, alterando o seu desempenho máximo.

Com relação ao efeito de bloco (laterais do galpão onde encontravam-se as gaiolas), este tornou-se indispensável na avaliação para as questões de temperatura corporal das aves, uma vez que esta pode influenciar diretamente no comportamento, desempenho e produção de ovos (Tabela 4). 
Tabela 4: Temperatura retal (TR), temperatura média corporal (TMC) e temperatura média da pele (TMP) das aves sob influência dos blocos

\begin{tabular}{cccc}
\hline TRATAMENTO & TR & TMP & TMC \\
\hline Lateral esquerda galpão & $41,157 \mathrm{a}$ & $31,988 \mathrm{a}$ & $38,406 \mathrm{a}$ \\
\hline Lateral direita galpão & $41,098 \mathrm{a}$ & $31,705 \mathrm{~b}$ & $38,280 \mathrm{~b}$ \\
\hline
\end{tabular}

Médias seguidas por letras distintas na coluna diferem entre si pelo teste Fisher a 5\% probabilidade

Não foi observado efeito dos blocos laterais na temperatura retal, porém houve um efeito significativo para blocos sobre as variáveis temperatura média da pele e temperatura média corporal. Esta diferença pode ser atribuída as condições de incidência solar direta nas aves durante determinados períodos

\section{CONCLUSÕES}

Conclui-se que para as condições experimentais empregadas no presente estudo não houve influência do enriquecimento ambiental sobre o comportamento das poedeiras, podendo esse resultado ter sido influenciado pelos horários de avaliação bem como pelas elevadas temperaturas e umidade

\section{RERÊNCIAS BIBLIOGRÁFICAS}

ABPA, Associação Brasileira de Proteína Animal, Relatório Anual, 2019. Disponível em:

http://abpabr.com.br/storage/files/3678c_final_ abpa_relatorio_anual_2019_portugues_web_re duzido.pdf>. Acesso em: 30 ago. 2019.

ABREU, V.M.N. ABREU, P.G.; COLDEBELLA, A.; GOMES, R.C.C.; AMARAL, A.G.; MORAES, S.P. Enriquecimento Ambiental de Gaiolas como Estratégia Prática para Incrementar o Bem-Estar e a Produção de Ovos de Poedeiras Pesadas. 1. ed. Concórdia, SC: EMBRAPA, 2006. 447 p. Disponível em: $<$ https://www.infoteca.cnptia.embrapa.br/bitstr eam/doc/443339/1/publicacaoz6b419o.pdf >. Acesso em: 18 jun. 2018.

ANDRADE, R.R.; TINÔCO, I. F. F.; BAÊTA, F. C.; BARBARI, M.; CONTI, L.; CECON, P. R.; CÂNDIDO, M.G.L.; MARTINS, I.T.A.; TELES JUNIOR, C. Evaluation of the surface do dia em uma das laterais do galpão.

Uma das formas da ave perder calor e por meio da evaporação (calor latente), que é realizada pela pele ou aparelho respiratório. Isto ocorre sobre influência da temperatura ambiente e umidade relativa do ar.

relativa do ar observadas durante o período experimental.

Quanto a qualidade dos ovos, observou-se melhor qualidade no aspecto da gravidade específica para o tratamento com enriquecimento ambiental das gaiolas.

temperature of laying hens in different thermal environments during the initial stage of age based on thermographic images. Agronomy Research, v. 15, n. 3, p. 629-638, 2017.

AZEVEDO, G.S.; SOUZA, J.P.L.; CARDOSO, J.A.; ARAÚJO, P.H.H.; NETA, E.R.S.; NOVAS, M.P.V. Produção de aves em sistema orgânico, PUBVET v.10, n.4, p.327-333, abr., 2016. Disponível em: < http://www.pubvet.com.br/artigo/2641/producc edilatildeo-de-aves-em-sistemaorgacircniconbsp>. Acesso em: 15 nov. 2016.

BARBOSA FILHO, J.A.D. Avaliação do BemEstar de Aves Poedeiras em Diferentes Sistemas de produção e condições Ambientais, utilizando Análise de Imagens. 2004. 110 p. Dissertação (Mestre em Agronomia) - Escola Superior de Agricultura "Luiz de Queiroz", Universidade de São Paulo, Piracicaba, 2004. Disponível em:<http://www.neambe.ufc.br/arquivos_publi 
cao/20111107113156.pdf>. Acesso em: 28 mai. 2018.

BARBOSA, O.; SILVA, R. Índice de Conforto Térmico para Ovinos, B. Indústr. Anim., N. Odessa, v.52, n.1. p.29-35, 1995. Acesso em: 28 nov. 2017.

CAMERINI, N.L., SILVA, R.C., NASCIMENTO, J.W.B., OLIVEIRA, D.L., and SOUZA, B.B. Surface temperature variation of laying hens created in two creation systems using Thermography. Agropecuária Científica no Semiárido, 12, 145-152. 2016.

CASTILHO, V. A. R.; GARCIA, R. G.; LIMA, N. D. S.; NUNES, K. C.; CALDARA, F. R.; NÄÄS, I. A., BARRETO, B., JACOB, F. G. Bem-estar de galinhas poedeiras em diferentes densidades de alojamento. Revista Brasileira de Engenharia de Biossistemas, v. 9, n. 2, p. 122-131. 2015.

FREITAS, E.R.; SAKOMURA, N.K.; GONZALEZ, M.M.; BARBOSA, N.A.A.B. Comparação de métodos de determinação da gravidade específica de ovos de poedeiras comerciais. Pesquisa Agropecuária Brasileira, Brasília-GO, v. 39, n. 5, p. 509-512, maio. 2004. Disponível em <http://www.scielo.br/pdf/\%0D/pab/v39n5/a14 v39n5.pdf $>$. Acesso em: 26 jun. 2018.

GUINZELLI,A.P.;BATTISTON,F.G.

Enriquecimento ambiental e avaliação do estresse de aves em relação ao desenvolvimento pós-nascimento. Unoesc Ciência-ACBS, v. 9, n. 1, p. 53-60, 2018.
MENEGALI, I. Avaliação de diferentes sistemas de ventilação mínima sobre a qualidade do ar e do desempenho de frangos de corte. 2009. 127f. Tese (Doutorado em Engenharia Agrícola) - Universidade Federal de Viçosa, Viçosa, 2009.

PEREIRA, DF.; BATISTA, E.S.; SANCHES, F.T.; GABRIEL FILHO, L.R.A.; BUENO, L.G.F. Comportamento de poedeiras criadas a diferentes densidades e tamanhos de grupo em ambiente enriquecido. Pesquisa Agropecuária Brasileira, Brasília-GO, v. 48, n. 6, p. 682-688, jun. 2013. Disponível em: $<$ http://dx.doi.org/10.1590/S0100-

204X2013000600014 >. Acesso em: 07 jun. 2018.

SANS,E.C.O.; FEDERICI, J.F.; HAMMERSCHMIDT,J.; CLEMENTE, H. C. ; DAHLKE, F. ;. O enriquecimento ambiental sobre o bem-estar de frangos de corte. Ciencia Rural, v. 44, p. 1867-1873, 2014

SOUSA, G. P.; PEREIRA, D. F.; RAMOS, D. D.; CATANEO, P. F.; WATANABE, K. Percepção dos produtores de ovos de Bastos/SP sobre ambiência, bem-estar animal e legislação na postura comercial. Energia na Agricultura 32: 40-47, 2017

YILDIRIM, M.; TASKIN, A. The effects of environmental enrichment on some physiological and behavioral parameters of broiler chicks. Brazilian Journal of Poultry Science, v. 19, n. 2, p. 355-362, 2017. 